\title{
Increasing the Consistency and Accuracy of Spectral Shearing Interferometry Via Multiple Shearing
}

\author{
Adam S. Wyatt ${ }^{1}$, Dane R. Austin ${ }^{1}$, Tobias Witting ${ }^{1}$, Ian \\ A. Walmsley ${ }^{1}$, Alexander Grün ${ }^{2}$, Philip K. Bates ${ }^{2}$, Olivier \\ Chalus $^{2}$ and Jens Biegert ${ }^{2,3}$ \\ ${ }^{1}$ Clarendon Laboratory, University of Oxford, Parks Road, Oxford, OX1 3PU, UK \\ ${ }^{2}$ ICFO-Insitut de Ciencies Fotoniques, Mediterranean Technology Park, 08860 \\ Castelldefels, Barcelona, Spain \\ ${ }^{3}$ ICREA Institucio Catalana de Recerca $i$ Estudis Avanc ats, 08010 Barcelona, \\ Spain \\ a.wyatt1@physics.ox.ac.uk
}

\begin{abstract}
We demonstrate improved accuracy and consistency for spectral-shearing interferometry using multiple shears. Using a new algorithm, different spectral shears are combined to perform accurate spectral phase measurements of complicated pulses from a hollow-core fiber system.

(C) 2008 Optical Society of America

OCIS codes: (320.7100) Ultrafast measurements, (120.5050) Phase measurement
\end{abstract}

\section{Introduction}

Few cycle near-infrared pulses are an extremely valuable tool in many areas of physics due to the ability to reach extremely high field strengths (e.g. $>10^{18} \mathrm{Wcm}^{-2}$ ), which is important in strong and ultra-high field physics. To date, there are only a few possible routes to generating these pulses, including hollow-core fiber or filamentation pulse compression, and optical parametric chirped pulse amplification. In all cases, the spectral intensity can be highly structure and/or the spectral phase can contain oscillations or be of higher order. The net result is that although it is possible to produce a main pulse with a duration on the order of a single to a few periods of the electric field, there also exists many 'low' intensity sub-pulses and pedestals. Even if these pulses contain less than a percentage of the main pulse energy, they can be strong enough to cause pre-ionization of a sample in ultra-high field applications (e.g. $>10^{13} \mathrm{Wcm}^{-2}$ ). Thus it is critical to be able to accurately measure these relatively-weak structures, which requires a measurement technique with a large dynamic range (i.e. the ratio between the peak and minimum intensity which can be measured in any given set of measurements).

Spectral phase interferometry for direct electric-field reconstruction (SPIDER)[1] is a specific form of spectral shearing interferometry. This technique enables the spectral phase of a test pulse to be measured by referring the spectral phase at one frequency with the spectral phase at another frequency separated from the first by the spectral shear, $\Omega$. In conventional SPIDER implementations, only single spectral shear is possible in addition to a calibration measurement (corresponding to a shear equal to zero). As the spectrometer resolution necessarily has to be able to resolve the spectral shear by several factors, the measured data contains redundant information that can allow the consistency of the measurement to be verified. However, this does not allow one to determine if there are any systematic errors, for example an error in the calibration of the fringe periodicity (or carrier phase), or the spectrometer itself. More advanced spectral shearing interferometers such as spatially encoded arrangement (SEA-)[2], zero additional phase (ZAP-)[3], long crystal (LX-)[4] SPIDERs and two dimensional shearing interferometry (2DSI)[5] enable multiple measurements to be taken at aribtrary shears. In all these cases, the carrier phase is independent from the spectral shear. Thus, measurements taken with different shears should only reproduce the same reconstructed spectral phase in the absence of any systematic errors.

Measurements of few-cycle pulses using SPIDER can also be improved via multiple shears. In the simplest concept, measurements with different sized shears is equivalent to measurements on different timescales. In the presence of noise, concatenation of the interferometric phase corresponding to a small shear, which is required to measure rapid phase variations, results in a relatively large error in the gross phase structure. A large shear reduces the number of sampling points and thus the error in the gross phase structure, but does not recover rapid phase variations. In addition, a large shear can enable one to bridge any holes in the spectrum (provided the different spectral regions are not well separated). 


\section{Experiment}

To highlight the advantages of performing spectral shearing with multiple shears, we have performed SEASPIDER measurements on a pulse which has been spectrally broadened in an argon filled hollow fiber, but without any external spectral phase compensation. Figure 1 shows the spectral intensity and the reconstructed spectral phase for two different shears and their combination. For each spectral shear, 9 measurements were taken to enable fluctuations to be measured. It is clear the the phase reconstructed from the small shear captures the fine structure, but errors accumulate in the gross structure, whereas the large shear reconstruction is the opposite - remaining accurate over the entire spectral range, but not recovering the fine spectral features. In the reconstructed phases for the combined case, the fine structure is measured without any errors in the gross structure.
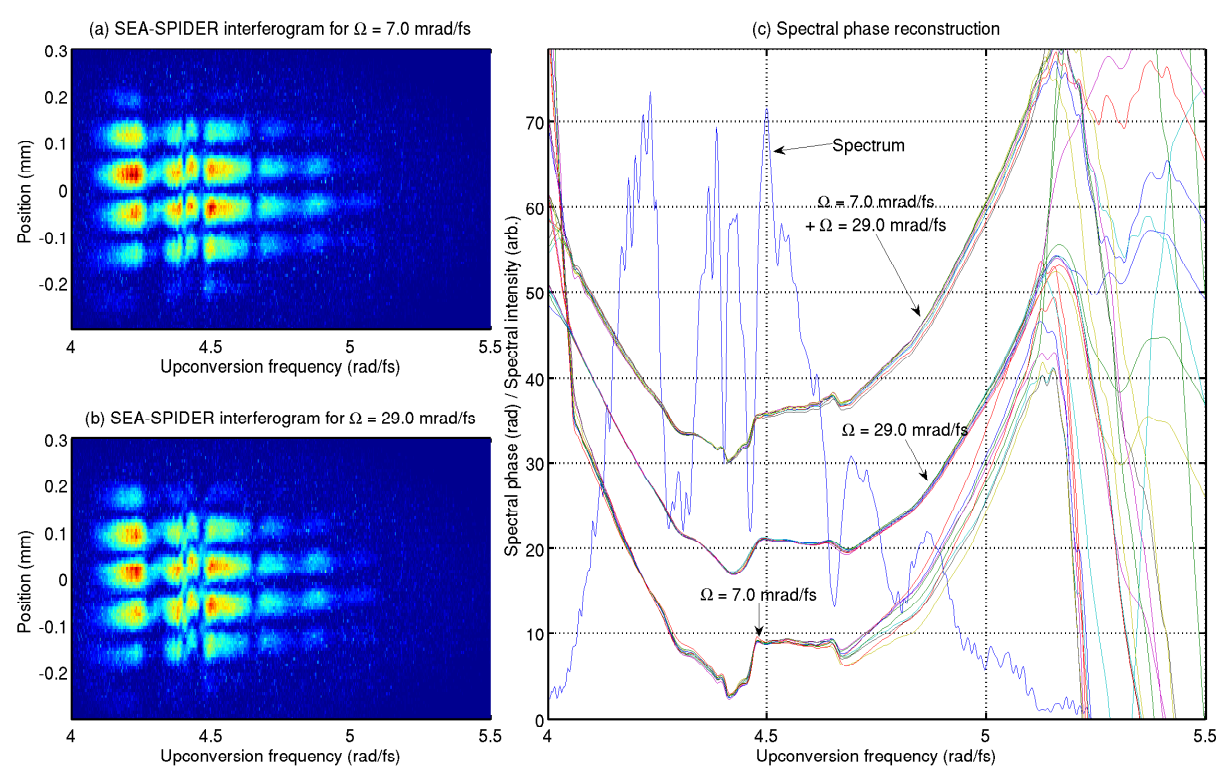

Fig. 1. Spectral intensity and reconstructed spectral phase from 9 different measurements using a spectral shear of: (bottom) $\Omega=7 \mathrm{mrad} / \mathrm{fs}(T \sim 900 \mathrm{fs}$ ); (middle) $\Omega=29 \mathrm{mrad} / \mathrm{fs}(T \sim 210 \mathrm{fs}$ ) and (top) combination of shears.

\section{Conclusion}

We have demonstrated that it is possible to increase the accuracy of spectral shearing interferometry by combining the interferometric phase from measurements made with a different shear. A combination of a large and small shear ensures accurate reconstruction over the entire spectral range and simultaneously recovering fine spectral features. Any systematic errors can be discovered by comparing the reconstruction from each individual single shear measurement. Thus it is clear that any spectral shearing device which can make measurements with different spectral shears without changing the carrier phase have an advantage over traditional SPIDER.

\section{References}

1. C. Iaconis and I. A. Walmsley, Opt. Lett., vol. 23, P792-794 (1998).

2. A. S. Wyatt, et al., Opt. Lett., vol. 31, P1914-1916 (2006).

3. P. Baum, et al., Opt. Lett., vol. 29, P210-212 (2004).

4. A. S. Radunsky, et al., Opt. Lett., vol. 31, P1008-1010 (2006).

5. J. Birge, et al., Opt. Lett., vol. 31, P2063-2065 (2006). 